\title{
USE OF WEB TECHNOLOGIES FOR RAISING AWARENESS ABOUT SEISMIC RISK
}

\author{
Ranjeet Joshi \\ Ramancharla Pradeep Kumar \\ Earthquake Engineering Research Centre \\ IIIT-H, Gachibowli, Hyderabad- 500032, \\ Andhra Pradesh, India \\ ranjeet@research.iiit.ac.in , \\ ramancharla@iiit.ac.in
}

\author{
Maluram Omprakash Sharma \\ Graduate Student, \\ Computer Aided Structural Engineering, \\ IIIT-H, Gachibowli, Hyderabad-500032, \\ Andhra Pradesh, India \\ maluram@students.iiit.ac.in
}

\begin{abstract}
Earthquake problems are not new to our country. We have witnessed several moderate earthquakes in last 2 decades causing innumerable property loss and life loss. In order to minimize the losses, we need to raise the awareness about earthquake resistant design of structures. This can be achieved by spreading the information on hazard, vulnerability and risk to concerned people.

Damage caused by a seismic event depends, to a large extent, on the a priori availability of information related to estimated hazard and vulnerability and their use in proper planning and construction. With the emergence of new web technologies such as Google Maps, Ruby on Rails and AJAX, systems which are robust in harnessing the collective information can be developed. In this paper, we discuss the quantification of risk of any location using these technologies.
\end{abstract}

\section{KEYWORDS}

Ruby on Rails, Google Map, Ajax

\section{INTRODUCTION}

No matter how quiet India appears in terms of seismic events, statistically last 2 decades were the most disastrous decades in terms of damage and loses caused by various seismic events. In just 5 years since 2001 the Andaman-Sumatra earthquake and Kashmir earthquakes have doubled the 100,000 people who were killed by earthquakes in the Indian region in the previous past 2 centuries.[1] Moreover many of these events like Uttarkashi earthquake (M6.6, 1991), Latur Osmanabad earthquake (M6.3, 1993), Jabalpur earthquake (M6.0, 1997), Chamoli earthquake (M6.8, 1999), Bhuj earthquake (M6.9, 2001) and Kashmir earthquake (M7.0, 2005) were of moderate to strong severity but the loss and damage caused by them were far beyond expected. The root cause for above mentioned earthquake disasters are lack of awareness about earthquakes and earthquake disasters, among general public in addition with lack of seismic risk information at the time of structural design. The basic aim of this application is to eliminate above mentioned fallacy by providing general public and professionals with adequate information on seismic risk.

On the other hand a joint research by the Internet and Mobile Association of India (IAMAI) and (IMRB) International suggests a significant rise in the number of Internet users in India - an increase from 33 million in March 2006 to 37 million in September 2006. It also suggests an increase in the number of active users from 21.1 Million in March 2006 to 25 Million in September 2006. Active Users are the users who have used the internet at least one in the last 30 days. It was also estimated that the Internet User base is likely to cross the 40 million mark by March 2007, with user base is likely to be 42 Million and the Active User base is 
likely to be 28 Million. These statistics indicates rapid growth of internet users in India [2].This application was developed with a vision of making an interactive system for disseminating crucial information like regional seismic risk to a large number of people through an easily accessible mode. And World Wide Web (WWW) was chosen as the most suitable platform for fulfilling our requirements of disseminating information instantly to large number of people.

\section{RISK EVALUATION}

Seismic Risk of an area is defined as the expected extent of damage to a property and loss of life at the time of a seismic event [3]. Thus seismic risk evaluation will be extremely useful in undertaking long-term mitigation and preparedness measures against seismic disasters. Risk assessment, on the other hand, requires data related to hazard, vulnerability, exposure time and economic activities. Thus, to fulfill these requirements thorough studies need to be undertaken to collect data and knowledge required for undertaking risk assessment and to utilize the power of IT in order to develop more interactive applications in the field of risk assessment. Seismic risk pertaining to any region depends upon 3 major components i.e. seismic hazard, seismic vulnerability and exposure time. Seismic hazard is defined in terms of ground motion parameter like Peak Ground Acceleration (PGA). According to IS 1893:2002, Indian subcontinent has been divided into 4 seismic zones based upon the expected PGA value in a particular region. This zonation based PGA has been used to represent the effects of seismic hazard in this application. Seismic Vulnerability on the other hand is defined as the degree of damage that is expected to occur depending on the type of structure in an area. Thus Earthquake Risk is the product of the hazard intensity and the vulnerability of buildings and the output of a seismic risk analysis could give the probability of damage and loss from nearby earthquake and its quantification requires socio economic and housing statistics [4].

\section{APPLICATION DEVELOPMENT}

This application was developed using Ruby on Rails (RoR), a full-stack framework for developing database-backed web applications according to the Model-View-Control (MVC) pattern [5]. The
MVC pattern is important because of its power of separating the data from the user interface. All the modules related to data goes into the model classes. All related to data - controlling functions goes into the controller class and the user interface module goes into the view class. This kind of architecture is useful in case of problems involving large amount of data with an acceptable variation with time as it separates both the functionalities from each other. Since the data pertaining to seismic risk evaluation involves large number of earthquake records with considerable randomness, Ruby on Rails became the best choice for developing this application. This separation of view from model and controller class also made mashing up of Google maps more easy and flexible. The overall process of application development can be classified into 3 categories:

1. Data collection and Model generation

2. Creating Controllers

3. View or HTML sheets design.

\subsection{Data Collection and Model Generation}

Data used in this application was a collective form of different input parameters required to compute seismic hazard and vulnerability. Hazard was estimated in terms of seismic zonation and corresponding peak ground acceleration (PGA).While vulnerability was defined in terms of type of structures and their risk for different seismic intensities.

\subsubsection{Seismic Hazard Estimation}

According to IS 1893: 2002 India has been classified into 4 seismic zones. These zones are different in terms of peak ground acceleration (PGA) values that are fixed for them as shown in Table 1[6]. Hazard Severity is classified different for different regions based upon these PGA values or zones. For the risk evaluation in our application we have classified the severities for different seismic zones as follows:

Table 1 Seismic Zones \&Corresponding Hazard Severity

\begin{tabular}{|c|c|c|}
\hline $\begin{array}{c}\text { Seismic } \\
\text { Zone }\end{array}$ & $\begin{array}{c}\text { Peak Ground } \\
\text { Acceleration (PGA) }\end{array}$ & Hazard Severity \\
\hline II & 0.1 & Low (L) \\
\hline III & 0.16 & Moderate (M) \\
\hline IV & 0.24 & High (H) \\
\hline V & 0.36 & Very High (VH) \\
\hline
\end{tabular}




\subsubsection{Seismic Vulnerability Estimation}

Vulnerability Severity Index for this application was taken from Vulnerability Atlas Map of India. This atlas was prepared by Building Materials and Technology Promotional Council (BMTPC) and the vulnerability severity is classified on the basis different types of structures under different seismic intensities. On the basis of predominant roof and wall materials, buildings were classified into 4 basic categories [7]:

Category - A

A1. Mud Wall - All roofs sloping

A2. Unburnt Brick - (1) Sloping roof, (2) Flat roof A3. Stone Wall - (1) Sloping roof, (2) Flat roof

Category - B

B. burnt Brick Wall - (1) Sloping roof, (2) Flat roof

Category - C

C1. Concrete Wall - (1) Sloping roof, (2) Flat roof

C2. Wood Wall - All roofs

C3. Ekra Wall - All roofs

Category $-\mathrm{X}$

X1. GI and other Metal sheets - All roofs

X2. Bamboo, Thatch, Grass, Leaves - All roofs

This classification was adopted from Census of Housing, Government of India (GOI), 1991.

\subsection{Creating Controllers}

Controllers were created to define methods operable on the stored data or the computed data. All logical aspect of this application was applied to the controller section.

\subsection{View or HTML Sheets Design}

This was the interface developed for user interactivity with the data. This was the most crucial part to develop as it involved amalgamation of various technologies for the betterment of application. Google maps were incorporated in order to visualize location based earthquakes. Overall view architecture was classified into following categories:

\subsubsection{Search Options}

Information related to earthquake records were classified on the basis of 4 prime parameters and their combinations as shown in Figure 1.

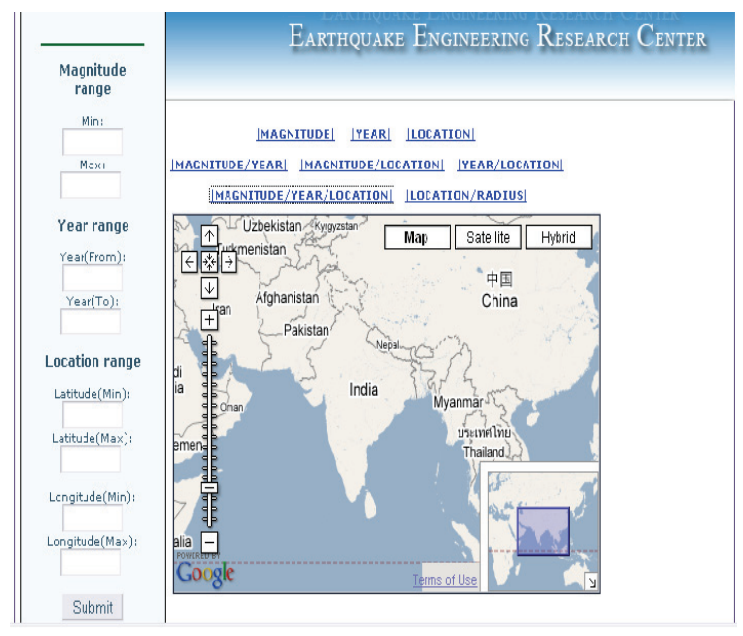

Figure 1 Front- End Search Options

These parameters were Magnitude, Year, Location and Radius. There combinations were formed by considering them together with remaining parameters (One by one and all together in some cases). The flexibility incorporated by this classification was the ease with which an end user can search the earthquake records based upon the ranges or limits incorporated to these parameters or their combination by the end user. Moreover the range selected from this view section goes to the controller where instructions were defined to mine out earthquakes those comes in the selected range and then to project these screened records onto the Google maps in the view section. This overall flow of information from Views to Controller to Models and again from Models to Controller to View makes this application more interactive and view more free from data dependency.

\subsubsection{Data Processing and Projection}

It is not always the case where information to the end user directly comes from the database. In most of the cases we have to process the information first and then only we can project it on the view for the end user. This fixes the interactions among the model, controller and view. For example in case of Seismic Risk evaluation, we have considered 2 prime factors for the risk index computation. These 
factors do not come directly from the database. Rather depending upon various other factors, these 2 risk evaluating factors get fixed somewhere in between before getting projected onto the View. Like in case of hazard computation input from the end user will be the location coordinates or the name of the region for which he wants to evaluate the seismic risk. The first task for this application was to evaluate the zone in which this location lies. This all computation took place in controller and finally the result obtained from the controller goes directly into the view. Here no controller - model interaction is involved.

\section{APPLICATION FEATURES}

For the hazard estimation, determination of seismic zone is required for the queried location. Latitude and Longitude were used as an input to define a queried location. This was made more interactive by using prototype, a java script library supplied free with rails. Problem with Google maps was its limitation of taking location info on the basis of location coordinates (latitude, longitude). But location coordinates are not the most used representation of location by common people. Most of the people identify their location either by name or by an associated event. Here we considered name based location identification as the most common trait and tried to implement a conversion code from location coordinates to location name in the controller method as shown in Figure 2.

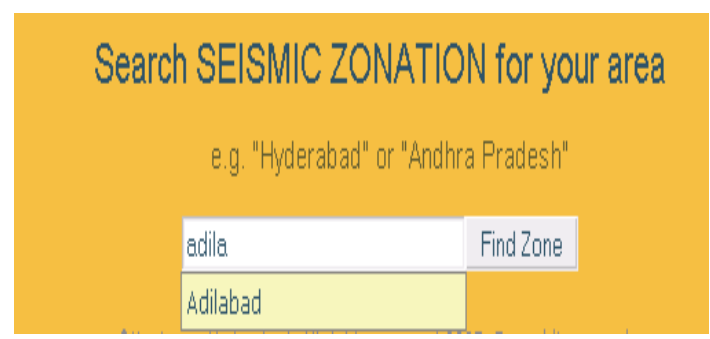

Figure 2 Seismic Zonation Search

This made application flexible in terms of input parameter and friendlier in terms of user's needs. On the other hand for vulnerability assessment, after fixing the location, one additional parameter i.e. type of houses is required. This necessitates estimation of types of construction in the locality of the queried location. So a feature was developed through which regional parameters could be queried using queried location as the centre and any radius ( $\mathrm{R})$ for which the region has to be defined. This location - radius based query for finding earthquakes in a radius of $500 \mathrm{Km}$ of Delhi region as shown in Figure 3.

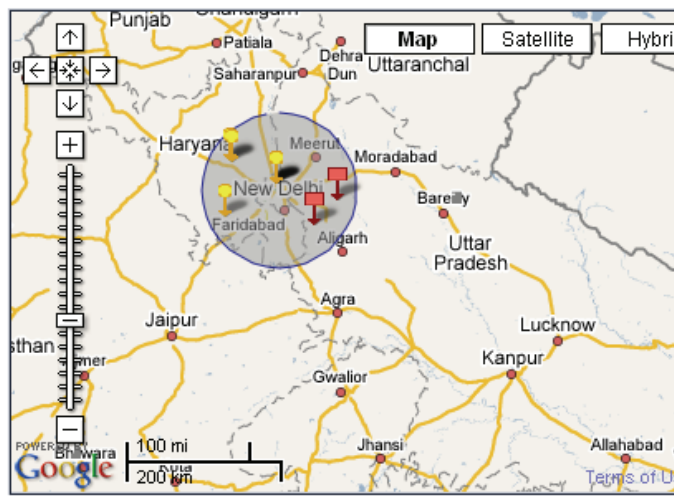

Figure 3 Location - Radius Based Search

\subsection{Additional Features}

Seismic Risk alone is not enough to mitigate the harms of a seismic disaster. The most important before or during a calamity is to share knowledge and information in the concerned community. This application was raised further into 4 different categories to fulfill this aspect of disaster mitigation. These categories are shown in Figure 4.

\section{Indian Earthquakes}

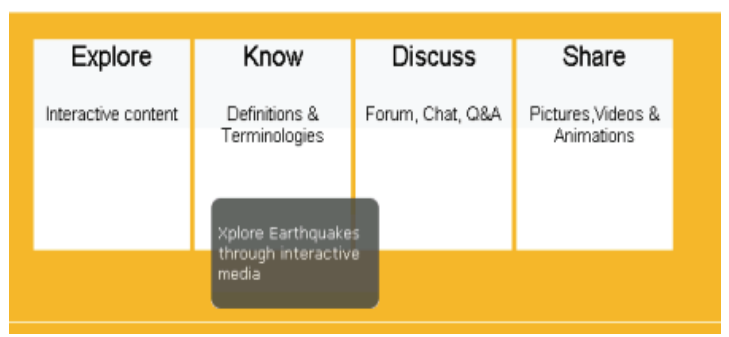

Figure 4 Four Major Categories for View

Explore section was kept for the interactive features of this application. Interactive features like those involved simple projection or computed results from the earthquake records. Know section was developed for the general information related to the terminologies and information related to the 
seismology. Discuss section was kept to make application more interactive in terms of end usage. This section was supplied with a forum, chat and a question answer segments in order to provide an online platform for discussing seismological termsand events. Share section was developed with a vision to make people share and download multimedia content related to seismology.

\section{RESULTS AND DISCUSSION}

So far application has been developed to incorporate hazard computation by identifying the seismic zone of the queried location. Database for vulnerability assessment is yet in a developing phase. Database for Indian earthquakes that struck in past 300 years was developed. Additional features like parameter based earthquake query, their count and related information display were incorporated. All the earthquakes and related information can now be visualized more thoroughly onto the Google maps as shown in Figure 5.

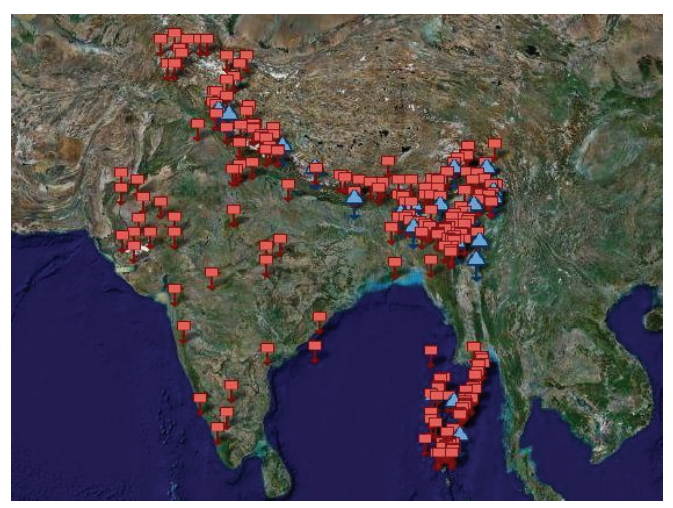

Figure 5 Projection of Earthquake on GoogleMap

\section{CONCLUSION}

As discussed in this paper, evaluation of seismic risk of an area is prerequisite for any planning and mitigation activities for seismic disasters and if it is available with us in advance than half the task of data collection is over. The application developed here will provide the seismic risk details based upon hazard and vulnerability of any particular region of the country. Thus providing significant information will help in planning and mitigating the seismic disaster along with sensitizing the general public through easily accessible and fast growing medium of web.

\section{REFERENCES}

[1] Roger Bilham, Background to the 1737 event. http://cires.colorado.edu/ bilham/1737Earthqu ake

[2] IAMAI-IMRB (2006), study pegs urban internet users at 36 million for September2006

[3] National Earthquake Management Strategies and Guidelines (NEMSAG) NDMA report 2006

[4] Anand S. Arya (2000). Recent developments toward earthquake risk reduction in India, Current Science, Vol. 79, No. 9, 10 November 2000

[5] http://www.rubyonrails.org/

[6] IS 1893:2002 (Part 1) Criteria for Earthquake Resistant Design of Structure

[7] Ujjwal Sur(2005), Vulnerability assessment of Building and population Related to Earthquake Hazard in Deharadun City Using Remote Sensing and Geoinformation Techniques . 